\title{
Acute Antibody-Mediated Rejection in Renal Transplantation: Current Clinical Management
}

\author{
Carrie Schinstock $\cdot$ Mark D. Stegall
}

Published online: 13 March 2014

(C) Springer International Publishing AG 2014

\begin{abstract}
Acute antibody-mediated rejection (AMR) is recognized as a major cause of graft loss in renal transplant recipients. Early acute AMR in the first few days after transplantation occurs primarily in sensitized renal transplant recipients with donor-specific alloantibody at the time of transplant and is a relatively "pure" form of acute AMR. Late acute AMR occurs months to years after transplantation and is commonly a mixed cellular and humoral rejection. While there is no consensus regarding optimum treatment, we contend that rational therapeutic approaches are emerging and that the acute episode can be managed in most instances. However, new therapies are needed to prevent ongoing chronic injury in these patients.
\end{abstract}

Keywords Antibody mediated rejection - Transplant glomerulopathy $\cdot$ Positive crossmatch transplantation $\cdot$ Renal transplantation $\cdot$ Kidney transplant

$\begin{array}{ll}\text { Abbreviations } \\ \text { AMR } & \text { Acute antibody-mediated rejection } \\ \text { DSA } & \text { Donor-specific antibody } \\ \text { MFI } & \text { Mean fluorescence intensity } \\ \text { PE } & \text { Plasmapheresis } \\ \text { IVIg } & \text { Intravenous immunoglobulin }\end{array}$

C. Schinstock $\cdot$ M. D. Stegall

William J. von Liebig Transplant Center, Mayo Clinic, Rochester, MN, USA

M. D. Stegall $(\bowtie)$

Division of Transplantation Surgery, Mayo Clinic, 200 First Street

SW, Rochester, MN 55905, USA

e-mail: stegall.mark@mayo.edu

\section{Introduction}

In the early days of renal transplantation, the major problem associated with donor-specific alloantibody (DSA) was hyperacute rejection. With more sensitive techniques to identify DSA and an increased understanding of the histological changes associated with DSA, it became clear that a form of acute rejection distinct from cellular rejection was possible in renal transplant recipients. This was termed "acute antibody mediated rejection" (AMR). As the rates of acute cellular rejection have fallen, acute antibody-mediated rejection has emerged as a major cause of graft loss in the weeks and months after transplantation.

This review examines our current understanding of acute AMR, including clinical management at our institution. We also discuss the possible connection between acute and chronic AMR and outline gaps in our understanding of both of these vexing entities. Finally, we examine specific therapeutic modalities and evidence of their utility in the management of acute AMR.

\section{Early and Late AMR: Two Unique Clinical Entities}

It is important to understand that there are actually two distinct clinical settings that are termed acute AMR, and their treatment may vary slightly (Table 1). The first is early AMR, which occurs in the first few days to weeks after transplantation. Early AMR occurs most commonly in allosensitized recipients (i.e., those with known DSA at the time of transplant), although it can occur rarely in patients with no DSA at transplant. The incidence varies with the amount of DSA present at the time of transplantation. In patients with high levels of DSA (i.e., sufficient to cause strongly positive crossmatch), the incidence in the first month after transplantation may be as high as $40 \%$, while the incidence in patients with a 
Table 1 Early versus late acute AMR

\begin{tabular}{|c|c|c|}
\hline & Early AMR & Late AMR \\
\hline Timing & Days to weeks post-transplant & Months to years post-transplant \\
\hline Pathophysiology & $\begin{array}{l}\text { Levels of preformed DSA increase from memory B-cell } \\
\text { response following antigen stimulation }\end{array}$ & $\begin{array}{l}\text { Formation of de novo DSA or increase in preformed DSA in setting of } \\
\text { suboptimal immunosuppression and/or concomitant cellular rejection }\end{array}$ \\
\hline Histology & $\begin{array}{l}\mathrm{C} 4 \mathrm{~d}+\text { peritubular capillaries on immunofluorescence, } \\
\text { acute tubular necrosis, peritubular capillaritis, and } \\
\text { glomerulitis }\end{array}$ & $\begin{array}{l}\text { Similar to early AMR in most cases: Peritubular capillaritis and } \\
\text { glomerulitis }+/- \text { C } 4 \text { d positivity in setting of interstitial inflammation } \\
\text { and tubulitis. Features of transplant glomerulopathy may also be } \\
\text { present. }\end{array}$ \\
\hline Treatment & $\begin{array}{l}\text { Plasmapheresis } \\
\text { IVIG } \\
\text { Eculizumab } \\
\text { Bortezomib } \\
\text { Rituximab }\end{array}$ & $\begin{array}{l}\text { Treatment of cellular rejection (e.g., steroids and anti-lymphocyte } \\
\text { therapy). } \\
\text { Consider plasmapheresis and IVIG if DSA MFI }>6000 \text {. In absence of } \\
\text { transplant glomerulopathy, eculizumab, bortezomib, or rituximab } \\
\text { could be considered. }\end{array}$ \\
\hline
\end{tabular}

negative crossmatch and DSA demonstrated only by solidphase assay is less than $10 \%$ [1].

Early AMR in this setting is relatively easy to identify, as it is usually a "purer" form in which cellular rejection is commonly absent. The recipient usually demonstrates a relatively rapid rise in serum creatinine level (normally day 10-14 after transplantation), and biopsy shows the classic signs of AMR, including $\mathrm{C} 4 \mathrm{~d}+$ staining of the peritubular capillaries on immunofluorescence and other features of injury such as acute tubular necrosis and microvascular inflammation (peritubular capillaritis and glomerulitis). More severe forms may show mesangiolysis and glomerular microthrombi. Serum levels of DSA are elevated due to a combination of preformed antibody and newly formed antibody from memory responses. In our series, a B flow cytometric crossmatch $>360$ (corresponding to a mean fluorescence intensity (MFI) of roughly 9000) in the early post-transplant period was almost always associated with an early AMR episode [2].

Early AMR can be quite severe and is a major cause of early graft loss. Therefore, we recommend aggressive early treatment in most cases. We would caution that while it is easy to attribute increases in serum creatinine to dehydration or an elevated tacrolimus level, in a highly sensitized patient 10 days after transplantation, early AMR should be the leading diagnosis. We may obtain the biopsy and draw the blood for serum DSA measurements, then begin plasma exchange (PE) therapy before these results return. In this way, we treat preemptively and can discontinue therapy if the diagnosis of AMR is not confirmed.

$\mathrm{PE}$ is our first line of therapy, and it is sufficient to decrease serum DSA levels to reverse most cases of early AMR (1). A course of 7 to 10 days of PE is normally required, and our goal is to reduce DSA levels to a B FXM $<200$ or an MFI $<4000$. Because the biopsy findings of AMR may persist for several days after the DSA levels have dropped, we tend to tailor therapy to DSA levels rather than the biopsy findings.
In more severe forms of early AMR, serum DSA levels and serum creatinine may continue to rise despite daily PE. These cases are at highest risk for graft loss and require more aggressive treatment. While there is no consensus in the field regarding the best treatment for these severe cases, we would add eculizumab (1200 mg initially, then $600 \mathrm{mg}$ after every $\mathrm{PE})$ to the treatment regimen. Terminal complement blockade with eculizumab appears to significantly block ongoing graft damage, and protects the graft until DSA levels begin to respond to PE [3•]. After 7 days of PE/eculizumab therapy, we reassess and may discontinue eculizumab if DSA levels are decreasing and if there is clinical improvement. As described below, other groups have advocated the use of splenectomy, bortezomib, rituximab and/or high-dose intravenous immunoglobulin (IVIg). Given the heterogeneity and rarity of these severe cases of early AMR, however, no controlled studies are available, and recommendations are based largely on a few cases.

In highly sensitized patients with an expected high incidence of early AMR, prevention may be a more prudent approach. For example, our group has shown that in patients with a B FXM channel shift $>200$ but less than 450 at baseline, the incidence of early AMR was $41 \%$ using a PE-based regimen [1]. When eculizumab was added to this regimen at the time of transplantation and continued for at least 1 month, the incidence of early AMR was only $7.7 \%$ [3•]. In addition, the few early AMR episodes that occurred were easily treated with $\mathrm{PE}$ and none required splenectomy. Eculizumab was able to be discontinued in half of the patients at 1 month because DSA levels remained low. Graft survival at 1 year was $100 \%$ in the eculizumab group versus $98 \%$ in the PE-alone group (unpublished).

While DSA levels will return to low levels (ex. MFI $<3000$ or so) in most patients after treatment of early AMR, some patients will have persistently high DSA levels. Despite having received intensive therapy and multiple PE treatments, these patients are at high risk for the development of chronic 
AMR and accelerated graft loss. There is likely no effective therapy for these patients. Our data suggest that continuation of eculizumab does not prevent chronic injury. Our current approach would be to discontinue therapy and then reassess at approximately 3 months after transplantation. At this point, if the patient shows evidence of chronic AMR (ex. microvascular inflammation and persistent DSA), we would treat with bortezomib $(1.3 \mathrm{mg} / \mathrm{BSA} \times 2$ cycles $[8$ doses] $)$, followed by $\mathrm{PE} \times 7$ days and then high-dose IVIg $(2 \mathrm{~g} / \mathrm{kg} \times 1)$. Unfortunately, this approach rarely results in dramatic improvement in either DSA levels or histology. New, more effective therapies are clearly needed.

\section{Late Acute AMR}

Acute AMR may also be diagnosed months to years after kidney transplantation. In this clinical setting, an acute elevation in serum creatinine develops, and a history of nonadherence to immunosuppression is commonly present. A biopsy shows that the Banff ' 97 histologic features associated with acute AMR $(\mathrm{C} 4 \mathrm{~d}+$ peritubular capillaries and evidence of histologic injury) and serum DSA level are present. Histologic features of acute cellular rejection (interstitial inflammation and tubulitis) may be present as well [4•]. In this setting, the production of DSA is likely due to the concomitant acute Tcell response to the allograft [5].

A critical decision that must be made in these patients is whether the acute AMR is truly contributing to graft dysfunction. In our program, we treat the cellular component of the late acute AMR with either corticosteroids or anti-T-cell antibody, depending upon the severity of the cellular rejection and the magnitude of graft dysfunction. If the DSA levels are relatively low (MFI $<2000$ or so), we may elect not to treat the antibody component of this rejection episode, as DSA levels may decrease when the T-cell rejection is treated.

In contrast, when DSA levels are high (MFI $>6000)$, we postulate that the DSA is truly contributing to graft dysfunction and merits treatment. We treat this episode similarly to an early acute AMR episode, employing multiple PE therapies as our first line of treatment. In contrast to early acute AMR, most cases of late acute AMR are mild and easily controlled by a combination of anti-T-cell therapy and PE. The major issue that quickly emerges is that graft function does not completely return to baseline, and evidence of chronic injury develops. Indeed, many patients have evidence of chronic injury (interstitial fibrosis and even transplant glomerulopathy) at the time of diagnosis of late acute AMR. In this respect, the distinction between acute and chronic injury is somewhat blurred. Again, the clinical setting dictates treatment based on our prediction of outcome. For example, a rapid rise in DSA with an allograft biopsy showing few chronic changes may be reversible, and thus may benefit from eculizumab therapy if
PE is unsuccessful. In contrast, a small rise in DSA with an allograft biopsy showing extensive chronic changes (e.g., Banff ' $97 \mathrm{cg} 3$ lesions) may not benefit from any therapy.

\section{Chronic AMR}

While a detailed discussion of chronic AMR is beyond the scope of this review, a few points regarding the role of acute AMR in chronic AMR deserve mention. First, the histology of acute and chronic AMR overlaps significantly, and it is unclear whether the pathobiology of the two are truly distinct. Although acute AMR has been shown to be a major risk factor for the development of chronic AMR, our recent eculizumab trial clearly shows that prevention of early AMR has little impact on the incidence of chronic AMR [3•] (Fig. 1). Thus, early clinical AMR may be critically dependent on C5, but chronic injury can occur without it. Antibody-mediated injury that does not require C5 may include direct activation of endothelial cells by DSA and microvascular inflammation (either vie c3a-mediated chemotaxis, recognition by the $\mathrm{Fc} \gamma$ receptor of macrophages and NK cells, or other mechanisms) $[6,7]$. We recently examined the long-term outcomes of these patients and found that persistently high DSA levels appeared to be the greatest risk factor for chronic injury.

Nonetheless, the development of acute AMR and how it is treated could affect the incidence of subsequent chronic AMR. Studies are needed to examine this link more clearly.

\section{Specific Therapies for Acute AMR}

Now that we have described some of the various clinical presentations of acute AMR and have presented our programmatic approaches, we believe that it is important to acknowledge that there are numerous other rational therapies for this

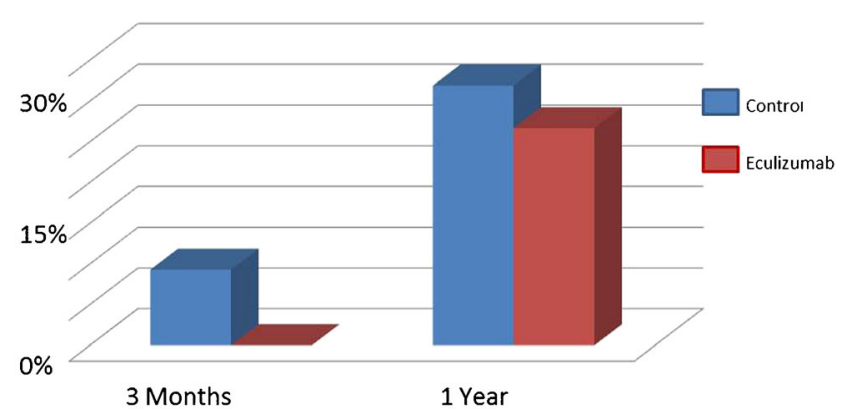

Fig. 1 Chronic AMR in the absence of acute AMR in eculizumab-treated patients. At 3 months post-transplant, no patients treated with eculizumab had transplant glomerulopathy (Banff cg score $>0$ ), while this was present in $9.3 \%$ of control patients. At 1 year, transplant glomerulopathy was present in $31.9 \%$ of control patients and $27.0 \%$ of eculizumab-treated patients $(p=0.62)$ 
issue (Table 2). We will discuss the details of each and any published data supporting their use.

Understanding the basic immunology of antibody production and antibody-mediated rejection is imperative in understanding the various treatment approaches for antibodymediated rejection and their limitations. In addition, an understanding of the basic biology of the rejection is essential for therapeutic development.

\section{Plasmapheresis}

Plasmapheresis, which is the physical removal of antibodies, was for many years the primary treatment modality for antibody-mediated rejection. The effect of plasmapheresis tends to be temporary, until the source of antibody is controlled. There is a marked variability in the efficacy of plasmapheresis, ranging from $0 \%$ to $93 \%$ [8]. Although randomized controlled trials have not confirmed a benefit from plasmapheresis alone [9], the therapy remains a first line of treatment for AMR.

Intravenous Immunoglobulin (IVIg)

Intravenous immunoglobulin (IVIg) is a blood product containing the pooled polyvalent IgG antibodies extracted from the plasma of over 1,000 donors and comes in a variety of formulations. It has been approved by the FDA for many autoimmune conditions and is FDA-approved for acute antibody-mediated rejection and desensitization for transplant recipients. While the precise mechanism of the immunomodulatory action of IVIg is largely unknown, IVIg is thought to saturate the Fc receptors on macrophages, suppress the production of inflammatory mediators, modulate complement, interfere with DSA binding and/or activity, and suppress idiotypic antibody [10].

To our knowledge, there are no published randomized controlled trials for use of IVIg in AMR. However, several groups have reported their experience using IVIg as treatment for acute AMR and as desensitization for highly sensitized transplant candidates [11-14]. Along with steroids and plasmapheresis, this therapy was the mainstay of treatment until newer agents such as rituximab became available. IVIg is currently often used as adjunctive therapy for AMR in combination with plasmapheresis and newer biological agents. The dose used for AMR and desensitization is typically higher than for immunodeficiency, at $1-2 \mathrm{mg} / \mathrm{kg}$ as a single dose or at multiple low-dose infusions of $100 \mathrm{mg} / \mathrm{kg}$ after plasmapheresis. IVIg is typically well-tolerated. High-dose therapy can sometimes lead to volume overload. Other complications include renal failure (typically from sucrose-containing solutions), headache, and thrombotic complications [15]. Additionally, because chromatographically derived IVIg products contain anti-B blood group antibodies, IVIg has been associated with temporary hemolytic anemia [16].

\section{Splenectomy}

Although it is an invasive treatment, splenectomy is an underappreciated potential therapy for acute antibody-mediated rejection and is associated with rapid recovery of renal function, especially in patients otherwise refractory to

Table 2 Therapeutic options for acute AMR

\begin{tabular}{|c|c|c|c|}
\hline Therapy & Studies & Mechanism of Action & Effectiveness \\
\hline Plasmapheresis & $\begin{array}{l}\text { Bonomini et al. (1985) [37] } \\
\text { Kirubakaran et al. (1981) [38] } \\
\text { Allen et al. (1983) [39] } \\
\text { Blake et al. (1999) [40] } \\
\text { Stegall et al. (2006) [41] }\end{array}$ & Physical removal of antibody & $\begin{array}{l}\text { Temporarily effective at reducing antibody } \\
\text { level depending on continued antibody } \\
\text { production. }\end{array}$ \\
\hline $\begin{array}{l}\text { Intravenous } \\
\text { Immunoglobulin } \\
\text { (IVIG) }\end{array}$ & $\begin{array}{l}\text { Glotz et al. (1993) [42] } \\
\text { Tyan et al. (1994) [43] } \\
\text { Jordan et al. (1998) [11] } \\
\text { Casadei et al. (2001) [13] } \\
\text { Tyan et al. (1994) [43] } \\
\text { Stegall et al. (2006) [41] } \\
\text { Lafaucheur et al. (2009) [44] }\end{array}$ & Multiple immunomodulatory actions & Variable efficacy \\
\hline Splenectomy & $\begin{array}{l}\text { Locke et al. (2007) [17] } \\
\text { Tzvetanov et al. (2012) [18] }\end{array}$ & Reduce B-cell and plasma cell burden & Variable efficacy \\
\hline Rituximab & $\begin{array}{l}\text { Kaposztas et al. (2009) [45] } \\
\text { Lefaucheur et al. (2009) [44] }\end{array}$ & $\begin{array}{l}\text { CD } 20+\text { inhibitor leading to reduction } \\
\text { in B-cells }\end{array}$ & Variable efficacy \\
\hline Bortezomib & $\begin{array}{l}\text { Everly et al. (2008) [26] } \\
\text { Walsh et al. (2010) [27] } \\
\text { Wong et al. (2010) [28] }\end{array}$ & $\begin{array}{l}\text { Proteasome inhibitor leads to plasma } \\
\text { cell apoptosis }\end{array}$ & Variable efficacy \\
\hline Eculizumab & Stegall et al. (2011) [3•] & Terminal complement (C5) inhibitor & $\begin{array}{l}\text { Very effective at inhibiting acute AMR, } \\
\text { but does not prevent chronic AMR. Expensive. }\end{array}$ \\
\hline
\end{tabular}


conventional treatments [17]. Six of eleven patients with acute AMR refractory to IVIg and plasmapheresis had improved renal function 2 weeks following splenectomy [18]. Normally the spleen is rich in mature B-cells and not plasma cells, but spleens removed at times of acute antibody-mediated rejection exhibit a distinct increase in CD138+ plasma cells as compared to spleens removed for other reasons [19]. One group found that in addition to increased CD138+ plasma cells the size of $\mathrm{T}$ and $\mathrm{B}$ lymphocyte aggregates were decreased [20]. Based on these findings, high antigenic burden may induce the spleen to sequester plasma cells or to enable rapid differentiation of B-cells [19]. Splenectomy should be considered in patients who are refractory to other therapies, especially as laparoscopic techniques and vaccination minimize surgical and infectious risks. The on future role of splenectomy in chronic antibody-mediated rejection is unclear.

\section{Rituximab}

Rituximab is a monoclonal antibody directed against CD20, a marker widely expressed on B-cells from the early pre-B-cell stage of development to the mature B-cell. Binding of the $\mathrm{Fc}$ portion of rituximab to $\mathrm{CD} 20$ leads to antibody-dependent cellular cytotoxicity, complement-dependent cytotoxicity, and a rapid $70-80 \%$ decline in the B-cell population. Other mechanisms of rituximab have been proposed in the rheumatology literature. Rituximab may downregulate CD40, the costimulatory molecule needed to augment the interaction between $\mathrm{T}$ and $\mathrm{B}-\mathrm{cells}$, or inhibit cytokines interfering with B-cell proliferation and differentiation [21].

In a nonrandomized retrospective study, patients treated with rituximab in addition to plasmapheresis and IVIg had improved graft and renal function compared to those treated with plasmapheresis and IVIg alone.

Unfortunately, the response to rituximab has been inconsistent. Some studies have shown significant benefit, while others are less encouraging. Rituximab is likely ineffective in circumstances of high antibody burden because the established short or long lived plasma cells producing the antibody lack CD20. The variable expression of CD20 on B-cells might also play a role in its variable efficacy [22].

\section{Bortezomib}

Inhibition of the plasma cell, with the ultimate goal of reducing antibody secretion, is a logical therapeutic step in treating antibody-mediated rejection. Bortezomib is a proteosome inhibitor that leads to apoptosis by inhibiting the degradation of abnormal and misfolded proteins. Proteasome inhibition has other immunomodulatory effects as well. Plasma cells are especially vulnerable to apoptosis with this therapy, and bortezomib has become one of the most common treatments for multiple myeloma. It has been theorized that high protein or antibody production in states such as multiple myeloma may increase susceptibility to proteasome inhibition [23]. Our group has shown that bortezomib causes apoptosis of human plasma cells in vitro, preventing alloantibody production [24]. This effect has not been demonstrated with the use of other major therapeutics in transplantation, including thymoglobulin, rituximab, and IVIg. In vivo, our group has also found that bortezomib leads to a reduction in plasma cells in the bone marrow of highly sensitized transplant candidates, although this did not translate into donor-specific antibody reduction [25]. However, it is possible that this therapy potentiates the effectiveness of plasmapheresis. We found a greater reduction in DSA levels with plasmapheresis in patients treated with bortezomib as compared to historical control patients treated with plasmapheresis alone [25].

Although there have been no randomized clinical trials using bortezomib for AMR, there have been several published clinical series. In the first such report, bortezomib alone was used to treat 8 episodes of AMR in 6 patients who were refractory to other common treatments, including plasmapheresis, IVIg, thymoglobulin, and rituximab. Treatment with bortezomib was associated with $>50 \%$ reduction in immunodominant DSA, stabilized to improved renal function, and improved renal histology [26]. This group also published their experience using bortezomib combined with plasmapheresis and rituximab in 2 patients as first-line therapy for early AMR. Both patients experienced a rapid reversal of AMR and elimination of DSA within 14 days [27]. The START (Strategic Antihumoral Therapies in Renal Transplantation) collaborative used a bortezomib-based regimen for AMR in 96 kidney, heart, and pancreas transplant recipients. The results, which have only been published in abstract form at this point, suggest that bortezomib may also be associated with a reduction in DSA.

Unfortunately, not all groups have experienced success with bortezomib therapy in treating AMR [28-31]. In 4 patients with subacute AMR and persistent DSA who were treated with 1 cycle of bortezomib, DSA levels were either unchanged or higher during follow-up of up to 270 days [29, 30]. Regardless of the variable efficacy of bortezomib, the therapy appears to be well-tolerated with 1-2 cycles of treatment [32]. The most common adverse effects include anemia, thrombocytopenia, peripheral neuropathy, and gastrointestinal problems.

The inconsistent results among studies of bortezomib are likely related to the complexity of AMR in general. As mentioned earlier, AMR exists as a spectrum of diseases, and it may or may not be associated with cellular rejection. The alloantibody-producing plasma cell population that leads to AMR may be very different in patients with late AMR in the setting of cellular rejection or noncompliance versus the early AMR that occurs as part of the memory response of a presensitized patient. In fact, greater reduction 
in immunodominant DSA after treatment with proteasome inhibition has been seen in early AMR as compared to late AMR [5].

The impact of proteasome inhibition may be related to the differential survival of the distinctive plasma cell populations, including the plasmablast, the short-lived plasma cell, and the long-lived plasma cell. The plasmablast is essentially a B-cell undergoing differentiation to ultimately become a short-lived plasma cell. These short-lived plasma cells are the most common of the antibody-producing cells and usually survive only a few days in the spleen or inflammatory sites at the time of immunization or infection [33]. The long-lived plasma cell is likely the most important with respect to long-term antibody production, and it is also the most difficult to eliminate. These non-dividing cells can survive for decades in special niches in the spleen or bone marrow, maintained by a delicate balance of cytokines and adhesion signals [33, 34].

Irrespective of the efficacy of bortezomib in treating AMR, targeting the plasma cell remains a focus for innovative therapies in both acute and chronic AMR. Other proteasome inhibitors have become available, and new therapies are being developed designed to interrupt the survival signals needed for the survival of the long-lived plasma cell.

\section{Eculizumab}

In the early 1990s, C4d staining of renal tissue was deemed reliable and saw increasing utilization in renal allograft patients. An inactive split product resulting from the cleavage of $\mathrm{C} 4 \mathrm{~b}, \mathrm{C} 4 \mathrm{~d}$ covalently binds to the site of complement activation, which is usually vascular endothelium in the renal allograft. With advances in tissue typing techniques, an association between donorspecific antibody and $\mathrm{C} 4 \mathrm{~d}$ staining was identified. Subsequently, several clinical studies have shown that positive peritubular capillary staining is associated not only with antibody-mediated damage but also with poor clinical outcomes. Although C4d-negative cases of acute antibody-mediated rejection exist [35], complement appeared to play a role in antibody-mediated damage. Yunnan-cobra venom factor (Y-CVF), a potential C3 inhibitor, has been shown to prevent acute antibodymediated damage in primates [36]. Eculizumab, a humanized monoclonal antibody against C5 that ultimately blocks the membrane attack complex, has been studied in humans [3•].

Our group treated 26 highly sensitized positive-crossmatch kidney transplant recipients with eculizumab at the time of transplant, weekly for the first 4 weeks, and monthly up to 1 year post-transplant, in patients with persistently high donorspecific antibody levels, and found a dramatic reduction in acute antibody-mediated rejection. The rate of antibodymediated rejection, as defined by high DSA, C4D positivity, and acute allograft dysfunction, developed in only $7.7 \%$ of patients as compared to $41.2 \%$ of historical controls [3•]. The protocol biopsy specimens with $\mathrm{C} 4 \mathrm{~d}$ positivity were in patients with high donor-specific antibody levels typically associated with acute allograft dysfunction. Given that 2 patients developed acute AMR despite complement blockade, a complement-independent mechanism of acute antibodymediated rejection likely exists. However, the results of this trial strongly suggest that complement plays a major role in mediating antibody damage in the transplanted kidney. A randomized multicenter open-label trial of eculizumab versus standard therapy is currently ongoing, and results are unavailable. The effect of eculizumab on chronic antibody-mediated rejection is largely unknown at this time.

Unfortunately, available treatments for antibody-mediated rejection have demonstrated varying levels of efficacy. While differences in patient populations and study design account for some of the variability in outcomes, differences also exist between patients with pre-formed antibody and those with antibody developed soon after transplant. In the highly sensitized positive-crossmatch population, long-lived terminally differentiated plasma cells that reside in the bone marrow and other secondary lymphoid tissue are difficult to target, and antibody is continually produced. In other situations, antibody production is the result of memory B-cell stimulation and rapid conversion to plasma cells.

\section{Conclusions}

The ability to diagnose and treat acute AMR is an essential skill for clinicians caring for renal transplant recipients today. Most cases of acute AMR can be reversed if treated promptly and aggressively. While we have outlined our programmatic approach, we also recognize that other therapies may be equally or even more effective, and we have tried to present these approaches here. We contend that while acute AMR is manageable, the major unmet need with respect to DSA is the prevention of chronic injury. A better understanding of the biology of chronic AMR and new therapeutic modalities are critical components in addressing this serious issue .

\section{Compliance with Ethics Guidelines}

Conflict of Interest Carrie Schinstock declares that she has no conflict of interest.

Mark D. Stegall reports grants from Alexion, grants from Millennium, and personal fees from iPerion.

Human and Animal Rights and Informed Consent This article does not contain any studies with human or animal subjects performed by any of the authors. 


\section{References}

Papers of particular interest, published recently, have been highlighted as:

- Of importance

1. Gloor JM et al. Baseline donor-specific antibody levels and outcomes in positive crossmatch kidney transplantation. Am J Transplant Off J Am Soc Transplant Am Soc Transplant Surg. 2010;10(3):582-9.

2. Burns JM et al. Alloantibody levels and acute humoral rejection early after positive crossmatch kidney transplantation. Am J Transplant Off J Am Soc Transplant Am Soc Transplant Surg. 2008;8(12):2684-94.

3. Stegall MD, et al. Terminal complement inhibition decreases antibody-mediated rejection in sensitized renal transplant recipients. Am J Transplant Off J Am Soc Transplant Am Soc Transplant Surg. 2011;11(11):2405-13. This study is important because it is the first large clinical series in using eculizumab to show dramatic reduction in acute antibody mediated rejection in positive crossmatch kidney transplant recipients.

4. Dorje C, et al. Early versus late acute antibody-mediated rejection in renal transplant recipients. Transplantation. 2013;96(1):79-84. Important study of the difference in outcomes between patients with early versus late acute antibody mediated rejection.

5. Walsh RC et al. Early and late acute antibody-mediated rejection differ immunologically and in response to proteasome inhibition. Transplantation. 2011;91(11):1218-26.

6. Zhang X, Reed EF. Effect of antibodies on endothelium. Am J Transplant Off J Am Soc Transplant Am Soc Transplant Surg. 2009;9(11):2459-65.

7. Lee CY et al. The involvement of FcR mechanisms in antibodymediated rejection. Transplantation. 2007;84(10):1324-34.

8. Gurland HJ, et al. Plasmapheresis in renal transplantation. Kidney Int Supp. 1983(14):S-82-4.

9. Roberts DM, Jiang SH, Chadban SJ. The treatment of acute antibody-mediated rejection in kidney transplant recipients - a systematic review. Transplantation. 2012;94(8):775-83.

10. Raghavaiah S, Stegall MD. New therapeutic approaches to antibody-mediated rejection in renal transplantation. Clin Pharmacol Ther. 2011;90(2):310-5.

11. Jordan SC et al. Posttransplant therapy using high-dose human immunoglobulin (intravenous gammaglobulin) to control acute humoral rejection in renal and cardiac allograft recipients and potential mechanism of action. Transplantation. 1998;66(6):800-5.

12. Jordan SC et al. Current approaches to treatment of antibodymediated rejection. Pediat Transplant. 2005;9(3):408-15.

13. Casadei DH et al. A randomized and prospective study comparing treatment with high-dose intravenous immunoglobulin with monoclonal antibodies for rescue of kidney grafts with steroid-resistant rejection. Transplantation. 2001;71(1):53-8.

14. Luke PP et al. Reversal of steroid- and anti-lymphocyte antibody-resistant rejection using intravenous immunoglobulin (IVIG) in renal transplant recipients. Transplantation. 2001;72(3):419-22.

15. Vo AA et al. Safety and adverse events profiles of intravenous gammaglobulin products used for immunomodulation: a singlecenter experience. Clin J Am Soc Nephrol. 2006;1(4):844-52.

16. Kahwaji $\mathrm{J}$ et al. Acute hemolysis after high-dose intravenous immunoglobulin therapy in highly HLA sensitized patients. Clin J Am Soc Nephrol. 2009;4(12):1993-7.
17. Locke JE et al. The utility of splenectomy as rescue treatment for severe acute antibody mediated rejection. Am J Transplant Off J Am Soc Transplant Am Soc Transplant Surg. 2007;7(4):842-6.

18. Tzvetanov I et al. The role of splenectomy in the setting of refractory humoral rejection after kidney transplantation. Transplant Proc. 2012;44(5):1254-8.

19. Kaplan B et al. Histopathology and immunophenotype of the spleen during acute antibody-mediated rejection. Am J Transplant Off J Am Soc Transplant Am Soc Transplant Surg. 2010;10(5):1316-20.

20. Tzvetanov I et al. Cell population in spleens during antibodymediated rejection: pathologic and clinical findings. Transplantation. 2012;94(3):255-62.

21. Tokunaga M et al. Down-regulation of CD40 and CD80 on B cells in patients with life-threatening systemic lupus erythematosus after successful treatment with rituximab. Rheumatology. 2005;44(2): 176-82.

22. Sanz I et al. Phenotypic and functional heterogeneity of human memory B cells. Semin Immunol. 2008;20(1):67-82.

23. Meister $\mathrm{S}$ et al. Extensive immunoglobulin production sensitizes myeloma cells for proteasome inhibition. Cancer Res. 2007;67(4): 1783-92.

24. Perry DK et al. Proteasome inhibition causes apoptosis of normal human plasma cells preventing alloantibody production. Am J Transplant Off J Am Soc Transplant Am Soc Transplant Surg. 2009;9(1):201-9.

25. Diwan TS et al. The impact of proteasome inhibition on alloantibody-producing plasma cells in vivo. Transplantation. 2011;91(5):536-41.

26. Everly MJ et al. Bortezomib provides effective therapy for antibody- and cell-mediated acute rejection. Transplantation. 2008;86(12):1754-61.

27. Walsh RC et al. Proteasome inhibitor-based primary therapy for antibody-mediated renal allograft rejection. Transplantation. 2010;89(3):277-84.

28. Wong $\mathrm{W}$, et al. Bortezomib in kidney transplant recipients with antibody mediated rejection: three case reports. Clin Transpl. 2009: $401-5$.

29. Sberro-Soussan R, et al. Bortezomib alone fails to decrease donor specific anti-HLA antibodies: 4 case reports. Clin Transpl. 2009: 433-8.

30. Sberro-Soussan R et al. Bortezomib as the sole post-renal transplantation desensitization agent does not decrease donor-specific anti-HLA antibodies. Am J Transplant Off J Am Soc Transplant Am Soc Transplant Surg. 2010;10(3):681-6.

31. Sberro-Soussan R, et al. Bortezomib alone fails to decrease donor specific anti-HLA antibodies: even after one year post-treatment. Clin Transpl. 2010:409-14.

32. Schmidt $\mathrm{N}$ et al. Prospective evaluation of the toxicity profile of proteasome inhibitor-based therapy in renal transplant candidates and recipients. Transplantation. 2012;94(4):352-61.

33. Hoyer BF et al. Short-lived plasmablasts and long-lived plasma cells contribute to chronic humoral autoimmunity in NZB/W mice. J Exp Med. 2004;199(11):1577-84.

34. Cassese $\mathrm{G}$ et al. Plasma cell survival is mediated by synergistic effects of cytokines and adhesion-dependent signals. J Immunol. 2003;171(4):1684-90.

35. Sis B, Halloran PF. Endothelial transcripts uncover a previously unknown phenotype: C4d-negative antibody-mediated rejection. Curr Opin Organ Transplant. 2010;15(1):42-8.

36. Chen Song $\mathrm{S}$ et al. Complement inhibition enables renal allograft accommodation and long-term engraftment in presensitized nonhuman primates. Am J Transplant Off J Am Soc Transplant Am Soc Transplant Surg. 2011;11(10):2057-66.

37. Bonomini $\mathrm{V}$ et al. Effects of plasmapheresis in renal transplant rejection. A controlled study. Trans Am Soc Artif Intern Organs. 1985;31:698-703. 
38. Kirubakaran MG et al. A controlled trial of plasmapheresis in the treatment of renal allograft rejection. Transplantation. 1981;32(2): $164-5$.

39. Allen NH et al. Plasma exchange in acute renal allograft rejection. A controlled trial. Transplantation. 1983;35(5):425-8.

40. Blake P, Sutton D, Cardella CJ. Plasma exchange in acute renal transplant rejection. Prog Clin Biol Res. 1990;337:249-52.

41. Stegall MD et al. A comparison of plasmapheresis versus high-dose IVIG desensitization in renal allograft recipients with high levels of donor specific alloantibody. Am J Transplant Off J Am Soc Transplant Am Soc Transplant Surg. 2006;6(2):346-51.

42. Glotz D et al. Suppression of HLA-specific alloantibodies by highdose intravenous immunoglobulins (IVIg). A potential tool for transplantation of immunized patients. Transplantation. 1993;56(2):335-7.

43. Tyan DB et al. Intravenous immunoglobulin suppression of HLA alloantibody in highly sensitized transplant candidates and transplantation with a histoincompatible organ. Transplantation. 1994;57(4):553-62.

44. Lefaucheur $\mathrm{C}$ et al. Comparison of combination plasmapheresis/ IVIg/anti-CD20 versus high-dose IVIg in the treatment of antibodymediated rejection. Am J Transplant Off J Am Soc Transplant Am Soc Transplant Surg. 2009;9(5):1099-107.

45. Kaposztas $Z$ et al. Impact of rituximab therapy for treatment of acute humoral rejection. Clin Transplant. 2009;23(1):6373. 